\title{
A method for estimating existence of pairwise STM transaction conflicts
}

\author{
Miroslav Popovic ${ }^{1, a}$, Branislav Kordic ${ }^{2}$, Marko Popovic ${ }^{1}$ and Ilija Basicevic ${ }^{1}$ \\ ${ }^{1}$ University of Novi Sad, Faculty of Technical Sciences, Trg Dositeja Obradovica 6, 21000 Novi Sad, Serbia
}

\begin{abstract}
STM transaction schedulers were introduced to improve system performance. However, designing online transaction scheduling algorithms is challenging because at the same time they should: (i) introduce minimal scheduling overhead, (ii) minimize the resulting makespan, and (iii) minimize contention in the resulting schedule. In our previous work we developed the online transaction scheduler architecture and the four scheduling algorithms, named RR, ETLB, AC, and AAC (listed in increasing order of their quality), for scheduling transactions on the Python STM. Both AC and AAC use Bernstein conditions to check for pairwise data races between transactions, at the cost of time complexity that is proportional to the product of the sizes of transaction's read and write sets, which may be significant. In this paper we propose a method for estimating existence of pairwise transaction conflicts whose time complexity is $\Theta(1)$. We validate this method by analysing the resulting transaction schedules for the three benchmark workloads, named RDW, CFW, and WDW. The result of this analysis is positive and encouraging - AAC using the new method produces the same result as when using Bernstein conditions. The limitation of the new method is that it may have false reports, both false negatives and false positives.
\end{abstract}

\section{Introduction}

Transactional Memory (TM) was originally introduced as an architectural support for lock-free concurrent data structures used on multicores [1]. By replacing traditional locks with TM transactions akin to database transactions, TM introduces higher level of abstraction, which both makes concurrent programming easier and enables composing new software components from existing building blocks [2]. These are the two main advantages of TM over locks.

Some theoretical foundations that enable analysis of TM programs have already been laid, which is important when engineering TM-based systems. For example, we may: (i) calculate parallelism using work-span method [3], (ii) determine conflict types and calculate their probabilities for some cases [4], and (iii) estimate transaction execution time based on log-normal distribution [5].

Right from its inception back in 1993, over the years, and to the present date, the TM was and continues to be the area of intensive research and development worldwide. Over this period, many TM implementations with various semantics and APIs were developed [2]. So nowadays, besides the hardware TMs (such as IBM's Blue Gene/Q, zEnterprise EC12, and Power 8 architectures, and Intel's Haswell architecture), there are various software TMs (STMs), hybrid TMs (HTMs), and distributed TMs (DTMs) [6]. In this paper, we use Python STM (PSTM) [7]. However, the main disadvantage of all these kinds of TMs is that their performance may be significantly degraded when they are exposed to high-contention workloads.

Transaction schedulers (aka contention managers) were introduced in order to mitigate this disadvantage. Transaction scheduling algorithms are widely studied in the literature. Some of the algorithms are theoretically well-established with known impossibility results and time complexity bounds $[8,9,10]$, whereas other heuristic algorithms are experimentally well-validated $[11,12]$. Still, there is a need for further research on online transaction scheduling algorithms.

Designing online transaction scheduling algorithms is challenging because at the same time they should: (i) introduce minimal scheduling overhead, (ii) minimize the resulting makespan, and (iii) minimize contention in the resulting schedule. In our previous work we developed the online transaction scheduler architecture and the four scheduling algorithms, named Round Robin (RR), Execution Time Load Balancing (ETLB), Avoid Conflicts (AC), and Advanced Avoid Conflicts (AAC), for scheduling transactions on the Python STM [13-14].

Both $\mathrm{AC}$ and AAC use Bernstein conditions [15] to check for pairwise data races between transactions, at the cost of time complexity that is proportional to the product of the sizes of transaction's read and write sets, which may be significant. In this paper we propose a method for estimating existence of pairwise transaction conflicts whose time complexity is $\Theta(1)$. We validate this method by analysing the resulting transaction schedules for the three benchmark workloads, named $\mathrm{RDW}, \mathrm{CFW}$, and WDW. The result of this analysis is

${ }^{a}$ Corresponding author: miroslav.popovic@rt-rk.uns.ac.rs 
positive and encouraging - AAC using the new method produces the same result as when using Bernstein conditions. The limitation of the new method is that it may have false reports, both false negatives and false positives.

The rest of the paper is organized as follows. Section 2 provides a brief overview of the scheduler architecture and the four scheduling algorithms (RR, ETLB, AC, and AAC). Section 3 presents the new method for estimating existence of pairwise STM transaction conflicts. Section 4 contains the analysis of the resulting transaction schedules for three workloads (RDW, CFW, and WDW) made for two workers. Section 5 presents conclusions.

\section{Overview of scheduling algorithms}

In this section, we first introduce necessary definitions, and then continue with a brief overview of the transaction scheduler architecture and the four transaction scheduling algorithms, namely RR, ETLB, $\mathrm{AC}$, and AAC.

A transaction $T$ is a pair $(f, V)$, where $f$ is a function executed by $T$, and $V$ is a pair $(R, W)$, where $R$ and $W$ are sets of t-variables that $T$ reads and writes, respectively. Let $t_{e}$ be the transaction execution time.

\subsection{Scheduler architecture}

The scheduler architecture consists of a single scheduler process $S$ and $n$ worker processes $W_{i}, i=1, \ldots, n$, which are running on $n$ processors (or cores). $S$ has its input queue $Q_{i n}$ of transactions, whereas each $W_{i}$ has its input queue $Q_{i}$ and its output queue $D_{i}$. Application processes enqueue new transactions to $Q_{i n}$.

$S$ operates in two alternating rounds. In the first round, $S$ dequeues transactions from $Q_{i n}$, one by one, until $Q_{\text {in }}$ becomes empty. For each dequeued $T, S$ calls the configured scheduling algorithm $A$ that returns the index $i$, which $S$ uses to enqueue $T$ to $Q i$. In the second round, $\mathrm{S}$ just waits for done signals at associated $D_{i}$ queues.

Each $W_{i}$ dequeues the next transaction $T_{j}=\left(f_{j}, V_{j}\right)$ from its $Q_{i}$ and executes it by calling $f_{j}$. If $T_{j}$ gets aborted, $W_{i}$ enqueues $T_{j}$ back to $Q_{i n}$. Once $Q_{i}$ becomes empty, $W_{i}$ enqueues the signal done to its $D_{i}$.

It should be noted that this scheduler architecture is implemented as a Python module, so it runs on top of the local OS (e.g. Windows/Linux). We assume that target hardware has enough cores, such that each $W_{i}$ runs on its own core; otherwise the local OS scheduler may compromise transaction schedules made by algorithm $A$.

\subsection{RR algorithm}

$\mathrm{RR}$ algorithm assumes that there is no information about a given $T$, i.e. that $T$ 's $t_{e}$ and $V$ are not known, so it just assigns $T$ to the next worker $W i$ in a circle. More precisely, the algorithm initializes its local counter $i$ to 0 , and then every time it is called, it calculates the next $i$ by incrementing it with modulo $n$, and returns the previous value of $i$. The scheduler in its turn uses $i$ to schedule the next transaction $T$.

The main advantage of RR algorithm is its minimal overhead, because its time complexity is $\Theta(1)$. On the other hand, RR algorithm is generally neither able to minimize makespan nor to minimize contention, simply because it takes the black-box approach and treats all the transaction equally.

\subsection{ETLB algorithm}

ETLB algorithm uses the method based on log-normal distribution [5] to estimate the transaction execution time $t_{e}$ for a given type of transaction. Additionally, the algorithm maintains the list of current worker's loads $L$, where $L_{i}$ is a cumulative execution time currently assigned to $W_{i}, i=1, \ldots, n$. Each time the new $T$ is assigned to $W_{i}$, the $T$ 's estimated $t_{e}$ is added to $L_{i}$.

The ETLB algorithm uses $L$ and the estimated $t_{e}$ of the next transaction $T$ from the input queue $Q_{i n}$ to greedily schedule $T$ to the least loaded worker process $W_{i}$, i.e. $i=\operatorname{index}(\min (L))$, where the function $\min$ returns $L_{i}$ and the function index returns $i$.

The main advantage of ETLB algorithm is that it effectively minimizes the makespan and maximizes the throughput. It does this at the expense of increased time complexity, which is $O(n)$, because the time complexity for the Python functions min and index over the list of size $n$ is $O(n)$ [16]. The main disadvantage of the algorithm is that the resulting schedule may have conflicts, because it assumes that the information about $\mathrm{t}$-variables used by transactions is not available. Maybe surprisingly, this means that even though the initial schedule has minimal makespan, actually it may carry high contention, and because of the conflicts, the final makespan may be even worst that for the RR algorithm.

\subsection{AC algorithm}

$\mathrm{AC}$ algorithm is an extended ETLB algorithm that also uses the information about t-variables used by each $T$, which is available in the pair $V=(R, W)$. In order to be able to detect and avoid conflicts among the next $T$ from $Q_{i n}$ and already scheduled transactions, the algorithm firstly introduces the notion of a scheduled transaction $F$, which is a transaction $T$ scheduled to start at $t_{b}$, where $F$ is a tuple $\left(t_{b}, t_{e}, V\right), t_{b}$ is $F$ 's start time, and $t_{e}$ is $F$ 's completion time. Secondly, for each $W_{i}$, the algorithm maintains the queue $Q F_{i}$ (implemented as a list) of scheduled transactions assigned to $W_{i}$.

Let $F_{1}=\left(t_{b 1}, t_{e 1}, V_{1}\right)$ be an already scheduled transaction from $Q F_{i}$ and $F_{2}=\left(t_{b 2}, t_{e 2}, V_{2}\right)$ be the next transaction from $Q_{i n}$ scheduled at $t_{b 2}$. The algorithm uses the method in Algorithm 1 to detect a possible pairwise conflict between $F_{1}$ and $F_{2} . F_{1}$ is attacking $F_{2}$ if it completes before $F_{2}$, because $F_{1}$ will get committed and $F_{2}$ will get aborted (line 4 ) and vice versa $F_{2}$ is attacking $F_{1}$ if it completes before $F_{1}$ (line 5). If none of $F \mathrm{~s}$ is attacking another $F$, there is no conflict (line 12). Otherwise, the method checks Bernstein conditions (lines 7-9), and if they are satisfied, meaning there is no 
data race, it concludes that there is no conflict (line 11). Otherwise, the conflict is detected (line 10).

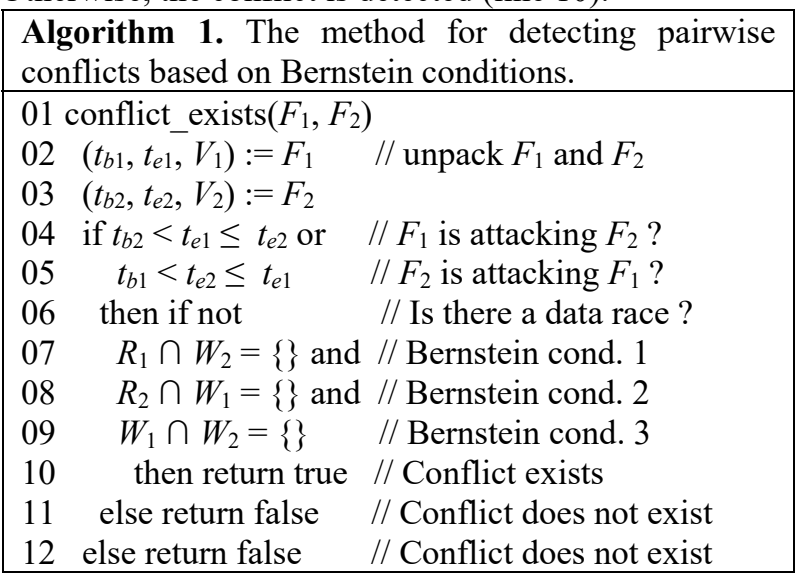

The time complexity of Algorithm 1 is proportional to the sum of the products of the sizes of the read and write sets checked in lines 7-9, i.e. $O\left(\left|R_{1}\right|\left|W_{2}\right|+\left|R_{2}\right|\left|W_{1}\right|+\right.$ $\left.\left|W_{1}\right|\left|W_{2}\right|\right)$. When all these sets are of equal size $m$, the time complexity is $O\left(\mathrm{~m}^{2}\right)$.

The AC algorithm, first determines the indexes of the least and most loaded workers, $i_{\min }$ and $i_{\max }$. Then it schedules $F_{2}$ at $L_{\text {min }}$, i.e. it sets $F_{2}$ to $\left(L_{\text {min }}, L_{\text {min }}+t_{e}, V_{2}\right)$, and checks whether there is a conflict among $F_{2}$ and any $F_{1}$ from all the $Q F_{i}, i=1, \ldots, n$ (it searches each $Q F_{i}$ in the backwards order starting from its end) using the method in Algorithm 1. If there is no conflict, the algorithm returns $i_{\text {min }}$. Otherwise, the algorithm conservatively back-offs by rescheduling $F_{2}$ to start at $L_{\max }$, i.e. it returns $i_{\max }$.

If we assume that $Q F_{i}$ lists are short (thus checking them may be done in almost constant time) and that all the read and write sets are of size $m$, the overall time complexity of the algorithm is $O\left(n \cdot m^{2}\right)$.

The main advantage of AC algorithm over the ETLB algorithm is that it is able to avoid conflicts, at the expense of increased time complexity of $O\left(n \cdot m^{2}\right)$. However, it is only capable of producing suboptimal schedules, because by using the conservative back-off after detecting a conflict at $L_{\min }$, it may miss a chance to find the next conflict-free position in time after $L_{\text {min }}$ and before $L_{\max }$, if such a position exists.

\subsection{AAC algorithm}

AAC algorithm is an extended $\mathrm{AC}$ algorithm that greedily searches for the optimal conflict-free position in time for the next transaction $T$, rather than giving up by making the conservative back-off when scheduling $T$ at $L_{\min }$ would create a conflict. In order to do this, the algorithm creates the sorted list of current worker loads, $L S=\operatorname{sorted}(L)$, and then checks all the time positions $L S_{i}$, $i=1, \ldots, n$, one by one, using the method in Algorithm 1, until it finds the first (earliest) $L S_{i}$ where scheduling $T$ would be conflict-free.

Under the assumption introduced in Section 2.4, the overall time complexity of the algorithm is $O\left(n^{2} \cdot m^{2}\right)$.

The main advantage of AAC algorithm over AC algorithm is that always produces optimal schedules, which are conflict-free and with the minimal makespan, but at the expense of more increased time complexity of $O\left(n^{2} \cdot m^{2}\right)$. The main motive of this paper is exactly to try to decrease the overall complexity to $O\left(n^{2}\right)$ by replacing the method in Algorithm 1 with the new method for estimating existence of pairwise conflicts in constant time $\Theta(1)$, as will be explained in Section 3.

\section{New method for estimating conflicts}

In this section, we present the new method for estimating existence of pairwise transaction conflicts whose time complexity is $\Theta(1)$, see Algorithm 2. The main idea of the method is to use the sizes of the read and write sets used by the pair of transactions to estimate the chance of a possible conflict, rather than to use the exact Bernstein conditions.

Intuitively, the smaller the size of these sets is, the smaller is the chance that there is a conflict. Of course, if both $W_{1}$ and $W_{2}$ are empty, then there is no conflict (line 7). Oppositely, if either $\left|R_{2}\right|$ or $\left|W_{1}\right|$ are greater than the parameter $r$ (line 8), and also symmetrically, if either $\left|R_{1}\right|$ or $\left|W_{2}\right|$ are greater than the parameter $r$ (line 9), then there is a significant chance that there is a conflict. Otherwise, the method assumes that the chance of a conflict is not significant (line 10).

The time complexity of Algorithm 2 is $\Theta(1)$, because the time complexity of the Python function len is $\Theta(1)$ [16].

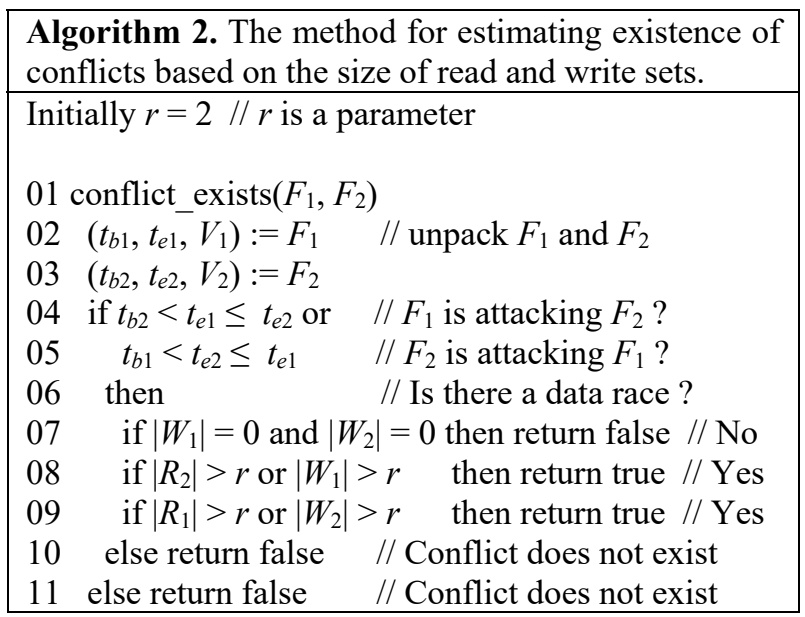

The main advantage of this method is that it is fast its time complexity is $\Theta(1)$. Of course, its main limitation is that it may have false reports. More precisely, in case when the conflict exists, but the sizes of read and write sets are smaller than $r$, the method will erroneously report false, i.e. a false negative will occur. Alternatively, in case when the conflict does not exist, but the sizes of read or write sets are greater than $r$, the method will erroneously report true, i.e. a false positive will occur.

Obviously, the result of Algorithm 2 depends on the characteristic of a given workload and on the value of the parameter $r$. The parameter $r$ was set to the value 2 in this paper, because it perfectly matches the three workloads (RDW, CFW, and WDW) that were used for 
the experimental validation of the scheduling algorithms in $[13,14]$, as will be shown in Section 4.

\section{Analysis of transaction schedules}

In this section we validate the new method by showing that transaction schedules made by AAC algorithm using the new method (Algorithm 2) are the same as transaction schedules made by AAC algorithm using the method based on Bernstein conditions (Algorithm 1). We do this for the three workloads, namely Read Dominated Workload (RDW), Conflict-Free Workload (CFW), and Write Dominated Workload (WDW), and for the scheduler architecture with two workers.
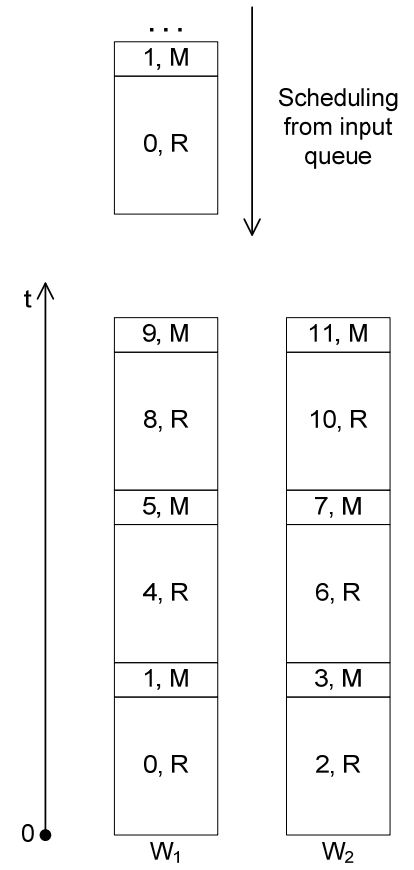

Figure 1. The transaction schedule for the workload RDW.

The above mentioned workloads are composed of the three kinds of transactions that are used in the PSTMbased program named Bank [13, 14]: (i) Read all accounts (R) which reads all t-variables representing accounts, (ii) Money transfer (M) which subtracts a given amount from the first t-variable (account) and adds it to the second, and (iii) Write all accounts (W) which initializes all the t-variables (accounts).

The workloads are packets of transactions whose indexes start from zero, which is here considered as an even number. In particular, RDW is the packet of R and $M$ transactions, such that even $(0,2, \ldots)$ transactions are $\mathrm{R}$ transactions and odd $(1,3, \ldots)$ transactions are $\mathrm{M}$ transactions. CFW is a packet of $\mathrm{M}$ transactions. WDW has the same structure as RDW with $\mathrm{W}$ transactions instead of R transactions. In all the workloads, all the $\mathrm{M}$ transactions are conflict-free among themselves.

We now analyse transaction schedules for each workload. In the following three figures, transactions are oriented vertically. Pairs in parenthesis indicate transaction's index and type - when we refer to a transaction we state both its index and type, e.g. zeroth $\mathrm{R}$ transaction, etc. $Q_{i n}$ is shown at the top, and input transactions are falling from it downwards, like in the evergreen game Tetris. The resulting schedule is shown at the bottom, in the form of two piles of transactions (one pile for each worker; the worker labels are shown below). The time axes points upwards, so the transactions at the bottom execute first.

Fig. 1 shows the resulting transaction schedule for the workload RDW. The schedule is periodic with the period of four transactions. Here is how this schedule is constructed. AAC algorithm using either method (either the method based on Bernstein condition or the new method) starts by scheduling the zeroth $\mathrm{R}$ transaction to $\mathrm{W}_{1}$. Then both methods report the conflict between the zeroth $\mathrm{R}$ and the first $\mathrm{M}$ transaction. The new method does this because $\left|R_{1}\right|$ is greater than $r$ (line 9 in Algorithm 2). Thus the first $\mathrm{M}$ transaction is scheduled to $\mathrm{W}_{1}$, too.

Then the second $\mathrm{R}$ transaction is scheduled to $\mathrm{W}_{2}$, because both methods report that resulting schedule is conflict-free. The new method does this because both write sets are empty (line 7 in Algorithm 2). Finally, the third $\mathrm{M}$ transaction is scheduled also to $\mathrm{W}_{2}$ because again both methods report that the result is conflict free. The new method does this because none of the sets are greater than $r$, so the method falls through the lines 7 to 9 and returns false (line 10). This completes the schedule period.
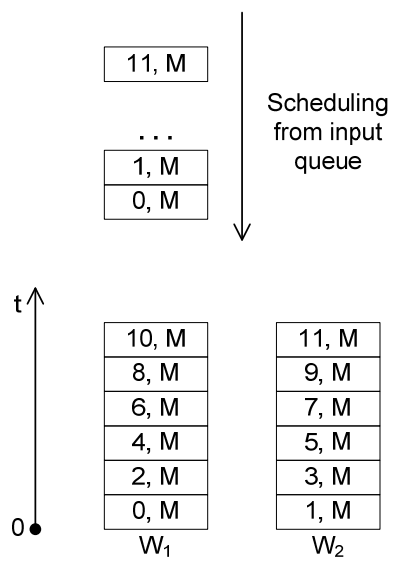

Figure 2. The transaction schedule for the workload CFW.

Fig. 2 shows the resulting transaction schedule for the workload CFW. The schedule is periodic with the period of two transactions. Here is how this schedule is constructed. AAC algorithm using either method starts by scheduling the zeroth $\mathrm{M}$ transaction to $\mathrm{W}_{1}$. Then both methods report that there is no conflict between the zeroth $\mathrm{M}$ and the first $\mathrm{M}$ transaction. The new method does this because none of the sets are greater than $r$, so the method returns false (line 10). Thus the first M transaction is scheduled to $\mathrm{W}_{2}$, and this completes the schedule period.

Fig. 3 shows the resulting transaction schedule for the workload WDW. The schedule is not really periodic but its construction follows the same patter for the group of four transactions. Here is how this schedule is constructed. AAC algorithm using either method starts by scheduling the zeroth $\mathrm{W}$ transaction to $\mathrm{W}_{1}$. Then both 
methods report the conflict between the zeroth $\mathrm{W}$ and the first $M$ transaction. The new method does this because $\left|W_{1}\right|$ is greater than $r$ (line 8 in Algorithm 2). Thus the first $M$ transaction is scheduled to $\mathrm{W}_{1}$, too.

Then the second $\mathrm{W}$ transaction is scheduled again to $\mathrm{W}_{1}$, because both methods report that conflict would exist between the zeroth $\mathrm{W}$ and the second $\mathrm{W}$ transaction if the latter would be scheduled to $\mathrm{W}_{2}$. The new method does this because $\left|W_{1}\right|$ is greater than $r$ (line 8 in Algorithm 2). Finally, the third $M$ transaction is scheduled also to $\mathrm{W}_{1}$ because again both methods report that conflict would exist between the zeroth $\mathrm{W}$ and the third $\mathrm{M}$ transaction if the latter would be scheduled to $\mathrm{W}_{2}$. The new method does this because $\left|W_{1}\right|$ is greater than $r$ (line 8 in Algorithm 2). This completes the schedule pattern. Effectively, the workload WDW is serialized to $\mathrm{W}_{1}$.
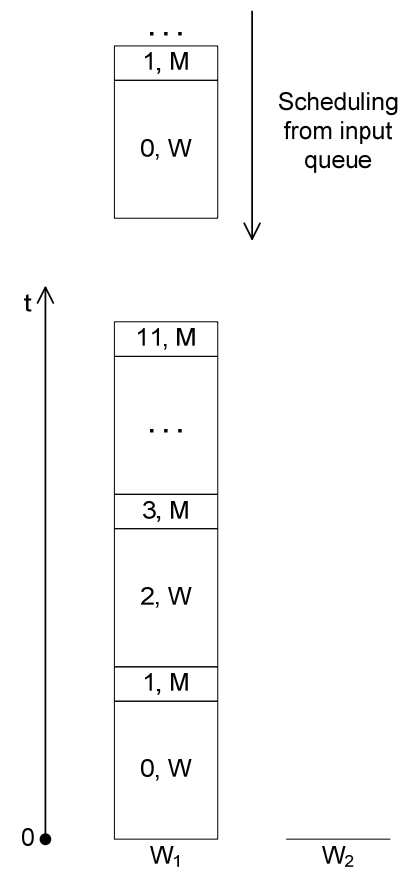

Figure 3. The transaction schedule for the workload WDW.

So, for all the workloads the new method based of the sizes of read and write sets provided the same reports as the method based on Bernstein condition. However, one should keep in mind that the new method could make mistakes on some other workloads.

\section{Conclusions}

In this paper, we propose a method for estimating existence of pairwise transaction conflicts whose time complexity is $\Theta(1)$. We validate this method by analysing the resulting transaction schedules for the three benchmark workloads, named RDW, CFW, and WDW.

The result of this analysis is positive and encouraging - AAC using the new method produces the same result as when it uses Bernstein conditions. The limitation of the new method is that it may have false reports, both false negatives and false positives.
In our future work, we plan to conduct experimental evaluation of the proposed method on a series of synthetic workloads and with more worker processes running on a manycore architecture, as well as to research other possible methods for estimating existence of pairwise transaction conflicts.

\section{Acknowledgment}

This work was partially supported by the Ministry of Education, Science and Technology Development of Republic of Serbia under Grant III-44009-2.

\section{References}

1. M. Herlihy, J.E.B. Moss, Transactional memory: architectural support for lockfree data structures, Proc. of the 20th Annual International Symposium on Computer Architecture, pp. 289-300 (1993)

2. T. Harris, J.R. Larus, R. Rajwar, Transactional Memory, 2nd edition (Morgan and Claypool, 2010)

3. M. Popovic, B. Kordic, I. Basicevic, Work, Span, and Parallelism of Transactional Memory Programs, Proceedings of the $4^{\text {th }}$ IEEE Eastern European Regional Conference on the Engineering of Computer Based Systems, pp. 59-66 (2015)

4. M. Popovic, I. Basicevic, Conflict Types and Probabilities in Stochastic TM-Programs, Proceedings of the 4th IEEE International Conference on Information Science and Technology, pp. 19-22 (2014)

5. M. Popovic, B. Kordic, I. Basicevic, Estimating Transaction Execution Times for a Software Transactional Memory, Proceedings of the 6th IEEE International Conference on Information Science and Technology, pp. 137-141 (2016)

6. P. Romano, R. Palmieri, F. Quaglia, L. Rodrigues, JCSS, 80, 1, pp. 257-276 (2014)

7. M. Popovic, B. Kordic, PSTM: Python Software Transactional Memory, Proceedings of the 22nd IEEE Telecommunications Forum, pp. 1106-1109 (2014)

8. R. Guerraoui, M. Herlihy, B. Pochon, Toward a theory of transactional contention managers, Proceedings of the 24th ACM Symposium on Principles of Distributed Computing, pp. 258-264 (2005)

9. H. Attiya, L. Epstein, H. Shachnai, Tami Tamir, Algorithmica, 57, 1, pp. 44-61 (2010)

10. H. Attiya, A. Milani, J Parallel Distrib Comput., 72, 10, pp. 1386-1396 (2012)

11. W.N. Scherer, M.L. Scott, Advanced contention management for dynamic software transactional memory, Proceedings of the 24th ACM Symposium on Principles of Distributed Computing, pp. 240248 (2005)

12. R.M. Yoo, H-H.S. Lee, Adaptive transaction scheduling for transactional memory systems", 
Proceedings of the 20th ACM Symposium on Parallelism in Algorithms and Architectures, pp. 169-178 (2008)

13. M. Popovic, B. Kordic, I. Basicevic, Transaction Scheduling for Software Transactional Memory, Proceedings of the 2nd IEEE International Conference on Cloud Computing and Big Data Analysis, pp. 191-195 (2017)

14. M. Popovic, B. Kordic, M. Popovic, I. Basicevic, Advanced algorithm for scheduling TM transactions with conflict avoidance, Proceedings of the 25nd IEEE Telecommunications Forum, pp. 844-847 (in Serbian, 2017)

15. A. J. Bernstein, IEEE Transactions on Electronic Computers, EC-15, 5, pp. 757-763 (1966)

16. Time Complexity page at python.org, https:/wiki.python.org/moin/TimeComplexity, visited on August 28 ${ }^{\text {th }}, 2018$ (2018) 